International Journal of Agriculture and Environmental Research

ISSN: 2455-6939

Volume: 07, Issue: 02 "March-April 2021"

\title{
ENERGY AND PROTEIN REQUIREMENTS OF LOCAL PRE-STARTER TURKEYS
}

\author{
${ }^{* 1}$ Ani, A. O.; ${ }^{1,2}$ Akuru, E. A.; ${ }^{1}$ Edeh, H.O.; ${ }^{2}$ Omeke J. N. \\ ${ }^{1}$ Department of Animal Science, University of Nigeria Nsukka 410001, Nigeria \\ ${ }^{2}$ Department of Livestock and Pasture Science, University of Fort Hare, Alice 5700, South Africa \\ *Corresponding author
}

DOI: https://doi.org/10.51193/IJAER.2021.7210

\begin{abstract}
The productivity of tropically bred local turkeys is often judged using the feeding standards of temperate birds. These standards are considered grossly inadequate in meeting the nutritional needs of the local turkey. Hence, this five-week study was conducted to determine the energy and protein requirements of local pre-starter turkeys in a hot humid tropical environment. One hundred and eighty day-old turkey poults were divided into 9 treatment groups with equal males and females. The birds were randomly assigned to 9 diets in a $3 \times 3$ factorial design with three crude protein (CP) $(24 \%, 26 \%$ and 28\%) and three energy (2600, 2700 and $2800 \mathrm{Kcal} / \mathrm{kg} \mathrm{ME})$ levels. Birds fed $2600 \mathrm{ME}$ diet had the highest final body weight (FBW), total weight gain (TWG), average daily weight gain (ADWG) and protein efficiency ratio (PER). The birds also consumed less feed to give an improved feed conversion ratio (FCR). Birds fed on $26 \% \mathrm{CP}$ diet had improved FBW, TWG, ADWG, and FCR and PER. The interaction of protein $(26 \% \mathrm{CP})$ and energy (2600 and $2700 \mathrm{ME}$ ) resulted in higher FBW, TWG, ADWG and better FCR. The interaction of $26 \% \mathrm{CP}$ and $2600 \mathrm{ME}$ also reduced serum aspartate aminotransferase, alanine aminotransferase, total protein, total bilirubin, and lactate dehydrogenase levels. The interaction of protein (24\% and 28\%) and energy (2800 and $2600 \mathrm{ME}$ ) also reduced urea and creatinine levels. Feeding local turkey poults on $26 \%$ crude protein and $2600 \mathrm{Kcal} / \mathrm{kg} \mathrm{ME}$ diets improved growth performance and did not impair their health status.
\end{abstract}

Keywords: Energy, protein, requirements, local turkeys, tropical environment, feeding standards. 
International Journal of Agriculture and Environmental Research

ISSN: 2455-6939

Volume: 07, Issue: 02 "March-April 2021"

\section{INTRODUCTION}

In recent times, the production of livestock has increased in developing countries. However, low protein intake has remained a major human nutritional problem in developing countries, such as Nigeria. This scenario is even worse for the non-wage and low income earners (1). Based on the recommendations of the World Health Organization (WHO), the best estimate for a population average requirement with regards to protein and amino acid requirement is $105 \mathrm{mg}$ nitrogen $/ \mathrm{kg}$ body/day weight or $0.66 \mathrm{~g}$ protein/ $\mathrm{kg}$ body weight/day (2). One strategy for addressing this problem is to improve the production of poultry species that have potentials for rapid growth rate and short generation interval (3). The management of poultry birds is usually not as hazardous and arduous as obtainable in livestock enterprises (4). Turkey production is one of the important sources of poultry meat supply $(5 ; 6)$. Despite the potentials in the supply of good quality animal protein and high rate of turnover on investment, Nigerians still consume small quantity of turkey when compared to the total animal protein consumed by an average Nigerian per day. This can be attributed to the low level of production and high cost of mature turkey (7). Hence, there is the need to enhance the production and productivity of the local turkey. However, the Nigerian local turkey is yet to attain its genetic potential, probably due to inadequate nutrition and issues associated with continuous selective breeding (8).More so, there are no established feeding standards that exist for these tropically bred local turkeys, whose productivity is often judged using the feeding standards of temperate raised birds. These standards are thus considered grossly inadequate for meeting the nutritional needs of the local turkey. For instance, during the first two months of life, starter and grower diets for exotic heavy-type growing turkeys should contain 28 and $26 \%$ crude protein (CP), respectively (9), which is in agreement with the breeder management guide recommendations (10). However, it is obvious that this feeding standard (as regards crude protein requirement) which is suitable for the temperate exotic turkeys is not suitable for the indigenous tropical turkeys due to differences in environmental conditions. Besides, there is a dearth of information on the energy and protein requirements of the Nigerian local turkeys at their various phases of growth. Against this backdrop, the current study was conducted to determine the energy and protein requirements of local turkey poults in a hot humid tropical environment.

\section{MATERIALS AND METHODS}

\section{Study location and duration}

This 35-d feeding trial was carried out at the Department of Animal Science Teaching and Research Farm, University of Nigeria, Nsukka. Nsukkaon the following coordinates 6.250N; $7.24^{\circ} \mathrm{E}$. All experimental procedures in the present study were performed according to the 
guidelines for the use of animals in biomedical research as described by the ethical research committee of the University of Nigeria, Nsukka.

\section{Experimental animals, housing and management}

A total of one hundred and eighty day-old turkey poults were randomly divided into nine experimental groups consisting of 20 turkey poults each. Each treatment group was balanced for their sexes (i.e. same number of males and females in each treatment). Poults in each treatment group were housed in a $2.6 \mathrm{~m} \times 3 \mathrm{~m}$ deep litter brooding pens with of fresh wood shavings that were changed when needed. The poultry house was an open-sided one with sides and demarcations between individual pens covered with wire gauze. Heat was provided by the use of kerosene stoves placed under metal hovers. The open sides were covered with thick polyethylene sheets during the brooding period of 4 weeks to conserve heat especially at night. Feed and water were provided to the birds ad libitum while additional light was provided at night using solar powered lamps to enable the birds eat at night. Dietary treatments were introduced from the onset of the feeding trial.

\section{Dietary treatments and chemical analysis}

Nine experimental diets were formulated in a $3 \times 3$ factorial arrangement involving three crude protein (CP) levels (24\%, 26\% and 28\%) and three energy levels (2600kcal $/ \mathrm{kg} \mathrm{ME,} 2700 \mathrm{kcal} / \mathrm{kg}$ $\mathrm{ME}$ and $2800 \mathrm{kcal} / \mathrm{kg} \mathrm{ME}$ ). The diets were designated thus; T1: $24 \% \mathrm{CP}+2600 \mathrm{ME}$; T2: $24 \%$ CP+ 2700 ME; T3: 24\% CP+ $2800 \mathrm{ME}$; T4: $26 \%$ CP+ 2600 ME; T5: 26\% CP+2700 ME; T6: 26\% CP+ $2800 \mathrm{ME}$; T7: $28 \% \mathrm{CP}+2600 \mathrm{ME}$; T8: $28 \% \mathrm{CP}+2700 \mathrm{ME}$; T9: $28 \% \mathrm{CP}+2800 \mathrm{ME}$. Representative samples of the diets were analysed to determine the proximate composition (Table 2) of crude protein, ether extract, crude fibre, ash according to the methods described by the Association of Official Analytical Chemists (2000). Table 1 shows the ingredients and nutrient composition of the basal diet, while the proximate composition of the diets is shown in Table 2. 
International Journal of Agriculture and Environmental Research

ISSN: 2455-6939

Volume: 07, Issue: 02 "March-April 2021"

Table 1: Ingredients and nutrient composition of basal diet

\begin{tabular}{|c|c|}
\hline Ingredients & Composition \\
\hline Maize & 33.00 \\
\hline Wheat offal & 15.00 \\
\hline Soybean meal & 12.00 \\
\hline Palm kernel cake & 10.00 \\
\hline Groundnut cake & 17.00 \\
\hline Fish meal & 5.00 \\
\hline Palm oil & 2.75 \\
\hline Bone meal & 0.25 \\
\hline Methionine & 0.25 \\
\hline Lysine & 0.25 \\
\hline Limestone & 4.00 \\
\hline Vitamin/mineral premix & 0.25 \\
\hline Salt & 0.25 \\
\hline \multicolumn{2}{|c|}{$\begin{array}{l}\text { a Vitamin+ mineral premix provided (per } \mathrm{kg} \text { of feed): } 8160 \mathrm{IU} \text { vit A, } 1700 \mathrm{IU} \text { vitamin D3, } 30.6 \mathrm{IU} \\
\text { vitamin E, } 2.7 \mathrm{mg} \text { vitamin K3 205mg vitamin B1, 2.03mg vitamin B2, 27.2mg niacin, } 10.2 \mathrm{mg} \text { calcium } \\
\text { pentothenate, } 2.02 \mathrm{mg} \text { vitamin B12, } 4.1 \mathrm{mg} \text { vitamin B6, } 1.7 \mathrm{mg} \text { folic acid, } 0.068 \mathrm{mg} \text { biotin, } 120 \mathrm{mg} \\
\text { ronozyme P500, } 350 \mathrm{mg} \text { choline, } 0.08 \mathrm{mg} \mathrm{I}, 0.34 \mathrm{mg} \mathrm{Co}, 0.2 \mathrm{mg} \mathrm{Se}, 70 \mathrm{mg} \mathrm{Mn,} 70 \mathrm{mg} \mathrm{Zn,} 6 \mathrm{mg} \mathrm{C} \text { and } \\
50 \mathrm{mg} \text { Fe NFF. Nitroge-free extract }\end{array}$} \\
\hline
\end{tabular}


International Journal of Agriculture and Environmental Research

ISSN: 2455-6939

Volume: 07, Issue: 02 "March-April 2021"

Table 2: Proximate composition of experimental diets

\begin{tabular}{llllllllll}
\hline Parameters & \multicolumn{7}{c}{ Dietary treatments } \\
\hline & T1 & T2 & T3 & T4 & T5 & T6 & T7 & T8 & T9 \\
\hline Dry matter & 92.90 & 92.90 & 91.60 & 90.95 & 92.55 & 94.05 & 92.70 & 95.40 & 92.80 \\
Ether extract & 2.00 & 2.10 & 1.80 & 1.90 & 2.55 & 1.85 & 3.55 & 2.90 & 3.35 \\
Crude protein & 24.52 & 23.20 & 23.95 & 27.21 & 27.58 & 26.77 & 28.46 & 28.85 & 27.76 \\
Crude fibre & 7.35 & 7.50 & 7.00 & 5.90 & 6.95 & 5.50 & 8.30 & 5.85 & 6.30 \\
Ash & 15.40 & 14.60 & 15.20 & 13.15 & 13.65 & 14.75 & 12.80 & 14.60 & 12.20 \\
NFE & 43.63 & 45.50 & 43.65 & 42.79 & 41.82 & 45.18 & 39.59 & 43.20 & 43.19 \\
\hline NFE: Nitrogen-free extract; T1: 24\% CP+ 2600 ME; T2: 24\% CP+ 2700 ME; T3: 24\% CP+ 2800 ME; T4: 26\% CP+ 2600 ME; T5: 26\% CP+ \\
2700 ME; T6: 26\% CP+ 2800 ME; T7: 28\% CP+ 2600 ME; T8: 28\% CP+ 2700 ME; T9: 28\% CP+ 2800 ME.
\end{tabular}

\section{Measurements}

\section{Growth performance}

The turkey poults were weighed at the beginning of the experiment, and thereafter on weekly basis to determine their weight gain. Fresh feed was offered to the birds every morning. Feed intake was then determined by subtracting the quantity of feed offered from the quantity of feed left over the following morning. Feed conversion ratio was calculated from the data on feed intake and weight gain as the number of grams of feed taken per gram of weight gained over the same period.

\section{Haematology and serum-biochemical analysis}

On the 35th day, about $4 \mathrm{ml}$ blood was collected from poults in each group using a syringe via vein puncture into well-labeled blood tubes. The first set of blood was collected into tubes containing ethylene diamine tetra acetic acid (EDTA) and used for the haematological evaluation of packed cell volume, haemoglobin concentration, white blood cell count and red blood count using the methods described by Jain (11). Mean cell haemoglobin haemoglobin concentration and mean cell haemoglobin concentration was calculated using the appropriate formulae. The 2nd set of blood was collected immediately placed into non-heparinized tubes. After that, the serum was isolated and stored at $-20^{\circ} \mathrm{C}$ and used to determine serum-biochemical indices such as creatinine, urea, total protein, aspartate amino transaminase, alanine amino transaminase, total bilirubin and lactate dehydrogenase using the Quimica Clinica Aplicada (QCA) test kits 
(Quimica Clinica Aplicada, Spain) and a Spectrum Lab 21A Spectrophotometer (Spectrum Lab, England) (12;13).

\section{STATISTICAL ANALYSIS}

The data obtained on growth performance, haematology and serum was analysed using the Stat Graphic Computer Package (14). Mean separation was done using Duncan's New Multiple Range Test option in SAS (14), and differences were declared significant at $\mathrm{p}<0.05$.

\section{RESULTS}

\section{Effect of varying dietary energy levels on the growth performance}

The effect of varying dietary levels of energyon the growth performances of local turkeys at the pre- starter phase is shown in Table 3. There were significant $(\mathrm{p}<0.05)$ differences in final body weight $(\mathrm{FBW})$, total weight gain (TWG), average daily weight gain (ADWG), total feed intake (TFI), average daily feed intake (ADFI), feed conversion ratio (FCR), protein efficiency ratio (PER) and feed cost (FC). Birds fed T1 (2600 ME) diet had significantly $(\mathrm{p}<0.05)$ higher FBW, TWG, and ADWG compared with the T2 (2700 ME) and T3 (2800 ME) birds. TFI and ADFI were highest $(\mathrm{p}<0.05)$ in T3 birds and lowest $(\mathrm{p}<0.05)$ in T1 birds. T1 birds had improved $(\mathrm{p}<0.05)$ FCR compared with the T2 and T3 birds. Birds fed T1 diet had the highest PER, whereas the lowest feed cost/kg gain values were recorded in $\mathrm{T} 3 \mathrm{birds}$.

Table 3: Growth performance of pre-starter local turkeys fed varying energy diets

\begin{tabular}{lllll}
\hline Parameters & T1 $(2600 \mathrm{ME})$ & T2 $(2700 \mathrm{ME})$ & T3 $(2800 \mathrm{ME})$ & SEM \\
\hline Initial body weight $(\mathrm{g})$ & 50.66 & 50.57 & 50.40 & 1.55 \\
Final body weight $(\mathrm{g})$ & $159.50^{\mathrm{a}}$ & $151.33^{\mathrm{b}}$ & $150.09^{\mathrm{b}}$ & 1.19 \\
Total weight gain $(\mathrm{g})$ & $108.84^{\mathrm{a}}$ & $100.77^{\mathrm{b}}$ & $99.68^{\mathrm{b}}$ & 1.19 \\
Av. daily WG $(\mathrm{g})$ & $3.88^{\mathrm{a}}$ & $3.59^{\mathrm{b}}$ & $3.56^{\mathrm{b}}$ & 0.04 \\
Total feed intake $(\mathrm{g})$ & $723.28^{\mathrm{c}}$ & $739.59^{\mathrm{b}}$ & $806.19^{\mathrm{a}}$ & 2.72 \\
Av. daily feed intake $(\mathrm{g})$ & $25.83^{\mathrm{c}}$ & $26.41^{\mathrm{b}}$ & $28.79^{\mathrm{a}}$ & 0.13 \\
Feed conversion ratio & $6.66^{\mathrm{b}}$ & $7.34^{\mathrm{a}}$ & $8.09^{\mathrm{a}}$ & 0.13 \\
Protein efficiency ratio & $0.15^{\mathrm{a}}$ & $0.14^{\mathrm{b}}$ & $0.14^{\mathrm{b}}$ & 0.15 \\
Feed cost/kg gain $(\mathrm{N})$ & $132.14^{\mathrm{c}}$ & $136.31^{\mathrm{b}}$ & $148.59^{\mathrm{a}}$ & 0.75
\end{tabular}
mean. 
International Journal of Agriculture and Environmental Research

ISSN: 2455-6939

Volume: 07, Issue: 02 "March-April 2021"

\section{Effect of varying dietary protein levels on growth performance}

The effect of dietary inclusion of varying protein levels on the growth performances of local turkeys at the pre- starter phase is shown in Table 4. The highest FBW, TWG, ADWG and PER were recorded in $\mathrm{T} 2$ birds (26\% CP diet) compared with the $\mathrm{T} 1$ (24\% CP diet) and $\mathrm{T} 3$ (28\% CP diet) birds. TFI, ADFI and feed cost were highest $(\mathrm{p}<0.05)$ in $\mathrm{T} 3$ birds and lowest in birds fed T1 diet. Birds fed T2 diet had improved ( $\mathrm{p}<0.05)$ FCR compared with the T1 and T3 birds.

Table 4: Growth performance of pre-starter local turkeys fed varying protein diets

\begin{tabular}{lllll}
\hline Parameters & T1 $(24 \%$ CP) & T2 $(26 \%$ CP $)$ & T3 $(28 \%$ CP $)$ & SEM \\
\hline Initial body weight $(\mathrm{g})$ & 50.54 & 50.59 & 50.50 & 1.54 \\
Final body weight $(\mathrm{g})$ & $143.51^{\mathrm{c}}$ & $169.21^{\mathrm{a}}$ & $148.20^{\mathrm{b}}$ & 1.19 \\
Total weight gain $(\mathrm{g})$ & $92.98^{\mathrm{c}}$ & $118.62^{\mathrm{a}}$ & $97.70^{\mathrm{b}}$ & 1.19 \\
Average daily weight gain $(\mathrm{g})$ & $3.32^{\mathrm{c}}$ & $4.23^{\mathrm{a}}$ & $3.49^{\mathrm{b}}$ & 0.04 \\
Total feed intake $(\mathrm{g})$ & $691.15^{\mathrm{c}}$ & $774.86^{\mathrm{b}}$ & $803.05^{\mathrm{a}}$ & 2.71 \\
Average daily feed intake $(\mathrm{g})$ & $24.68^{\mathrm{c}}$ & $27.67^{\mathrm{b}}$ & $28.68^{\mathrm{a}}$ & 0.13 \\
Feed conversion ratio & $7.43^{\mathrm{b}}$ & $6.54^{\mathrm{c}}$ & $8.22^{\mathrm{a}}$ & 0.28 \\
Protein efficiency ratio & $0.14^{\mathrm{b}}$ & $0.163^{\mathrm{a}}$ & $0.13^{\mathrm{c}}$ & 0.00 \\
Feed cost/kg gain $(\mathrm{N})$ & $128.28^{\mathrm{c}}$ & $161.10^{\mathrm{b}}$ & $178.45^{\mathrm{a}}$ & 0.74
\end{tabular}

a,b, c Means within columns with different superscripts differ significantly $(\mathrm{P}<0.05)$; WG: Weight gain; Av.: Average;SEM: Standard error of mean.

\section{Interaction effects of varying dietary protein and energy on growth performance}

The interaction effects of varying dietary levels of protein and energy on the performance of turkey poults is shown in Table 5. FBW, TWG and ADWG were highest $(\mathrm{p}<0.05)$ in T4 $(26 \% \mathrm{CP}$ + 2600ME) and T5 (26\% CP + $2700 \mathrm{ME})$ birds, and lowest (p<0.05) in T2 (24\% CP+2600 ME) birds. TFI and ADFI were highest $(\mathrm{p}<0.05)$ in birds fed $\mathrm{T} 3(24 \% \mathrm{CP}+2800 \mathrm{ME})$ and $\mathrm{T} 8$ $(28 \% \mathrm{CP}+2700 \mathrm{ME})$ diets and lowest $(\mathrm{p}<0.05)$ in $\mathrm{T} 1(24 \% \mathrm{CP}+2600 \mathrm{ME})$ and $\mathrm{T} 2$. FCR was improved in T1 and T5 birds compared with birds fed T2, T3, T7 (28\% CP + $2600 \mathrm{ME})$, T8 and T9 $(28 \% \mathrm{CP}+2800 \mathrm{ME})$ diets. PER was highest $(\mathrm{p}<0.05)$ in T5 birds and lowest $(\mathrm{p}<0.05)$ in those fed $\mathrm{T} 2$ diet. The lowest $(\mathrm{p}<0.05)$ feed cost $/ \mathrm{kg}$ gain values were recorded for $\mathrm{T} 1$ and $\mathrm{T} 2$ birds. 
International Journal of Agriculture and Environmental Research

ISSN: 2455-6939

Volume: 07, Issue: 02 "March-April 2021"

Table 5: The interaction effect of varying dietary protein and energy levels on the growth performance of pre-starter local turkeys

\begin{tabular}{lllllllllll}
\hline Parameters & T1 & T2 & T3 & T4 & T5 & T6 & T7 & T8 & T9 & SEM \\
\hline Initial BW & 50.54 & 50.67 & 50.40 & 50.73 & 50.57 & 50.47 & 50.70 & 50.47 & 50.33 & 0.27 \\
Final BW & $155.50^{\mathrm{bc}}$ & $126.67^{\mathrm{e}}$ & $148.37^{\mathrm{cd}}$ & $172.60^{\mathrm{a}}$ & $173.67^{\mathrm{a}}$ & $161.37^{\mathrm{b}}$ & $150.40^{\mathrm{cd}}$ & $153.67^{\mathrm{bc}}$ & $140.53^{\mathrm{d}}$ & 2.07 \\
Total WG & $104.96^{\mathrm{bc}}$ & $76.00^{\mathrm{e}}$ & $97.97^{\mathrm{cd}}$ & $121.87^{\mathrm{a}}$ & $123.10^{\mathrm{a}}$ & $110.90^{\mathrm{b}}$ & $99.70^{\mathrm{cd}}$ & $103.20^{\mathrm{bc}}$ & $90.20^{\mathrm{d}}$ & 2.06 \\
ADWG & $3.75^{\mathrm{bc}}$ & $2.71^{\mathrm{e}}$ & $3.50^{\mathrm{cd}}$ & $4.34^{\mathrm{a}}$ & $4.39^{\mathrm{a}}$ & $3.96^{\mathrm{b}}$ & $3.56^{\mathrm{cd}}$ & $3.69^{\mathrm{bc}}$ & $3.22^{\mathrm{d}}$ & 0.07 \\
Total FI & $599.64^{\mathrm{d}}$ & $610.44^{\mathrm{d}}$ & $863.38^{\mathrm{a}}$ & $814.71^{\mathrm{b}}$ & $759.28^{\mathrm{c}}$ & $750.57^{\mathrm{c}}$ & $755.47^{\mathrm{c}}$ & $849.06^{\mathrm{a}}$ & $804.61^{\mathrm{b}}$ & 3.44 \\
ADFI & $21.42^{\mathrm{d}}$ & $21.80^{\mathrm{d}}$ & $30.84^{\mathrm{a}}$ & $29.09^{\mathrm{b}}$ & $27.12^{\mathrm{c}}$ & $26.81^{\mathrm{c}}$ & $26.98^{\mathrm{c}}$ & $30.32^{\mathrm{a}}$ & $28.74^{\mathrm{b}}$ & 0.23 \\
FCR & $5.72^{\mathrm{d}}$ & $8.04^{\mathrm{b}}$ & $8.81^{\mathrm{ab}}$ & $6.70^{\mathrm{cd}}$ & $6.17^{\mathrm{d}}$ & $6.77^{\mathrm{cd}}$ & $7.59^{\mathrm{abc}}$ & $8.23^{\mathrm{ba}}$ & $8.92^{\mathrm{a}}$ & 0.22 \\
PER & $0.16^{\mathrm{abc}}$ & $0.11^{\mathrm{e}}$ & $0.15^{\mathrm{c}}$ & $0.17^{\mathrm{ab}}$ & $0.17^{\mathrm{a}}$ & $0.15^{\mathrm{bc}}$ & $0.13^{\mathrm{d}}$ & $0.13^{\mathrm{d}}$ & $0.12^{\mathrm{de}}$ & 0.00 \\
$\begin{array}{l}\text { Feed } \\
\text { cost/kg } \\
\text { gain }\end{array}$ & $109.56^{\mathrm{g}}$ & $112.02^{\mathrm{g}}$ & $159.13^{\mathrm{e}}$ & $169.39^{\mathrm{c}}$ & $158.47^{\mathrm{f}}$ & $157.25^{\mathrm{f}}$ & $167.88^{\mathrm{d}}$ & $189.36^{\mathrm{a}}$ & $180.09^{\mathrm{b}}$ & 1.29 \\
& & & & & & & & & &
\end{tabular}

a,b, c Means within columns with different superscripts differ significantly (P<0.05); BW: Body weight; WG: Weight gain; ADWG: Average daily weight gain; FI: Feed intake; ADFI; Average daily feed intake; FCR: Feed conversion ratio; PER; Protein efficiency ratio; T1: $24 \%$ CP+ $2600 \mathrm{ME}$; T2: 24\% CP+ $2700 \mathrm{ME}$; T3: 24\% CP+ $2800 \mathrm{ME}$; T4: 26\% CP+ $2600 \mathrm{ME}$; T5: 26\% CP+ 2700 ME; T6: 26\% CP+ 2800 ME; T7: 28\% CP+ 2600 ME; T8: $28 \%$ CP+ 2700 ME; T9: $28 \%$ CP+ 2800 ME.SEM: Standard error of mean.

\section{Effect of varying dietary energy levels on haematology and serum-biochemical indices}

As shown in Table 6, dietary treatments had significant $(\mathrm{p}<0.05)$ effect on the mean cell haemoglobin concentration (MCHC) and all serum parameters evaluated. MCHC was highest $(\mathrm{p}<0.05)$ in T2 birds $(2700 \mathrm{ME})$ and lowest $(\mathrm{p}<0.05)$ in birds fed T3 (2800 ME) diet. Creatinine and urea levels were highest $(\mathrm{p}<0.05)$ in $\mathrm{T} 1(2600 \mathrm{ME})$ and lowest $(\mathrm{p}<0.05)$ in T3 birds. Aspartate transaminase (AST) and alanine transaminase (ALT) levels were highest $(\mathrm{p}<0.05)$ in $\mathrm{T} 2$ and T3 birds and lowest $(\mathrm{p}<0.05)$ in $\mathrm{T} 1$. Birds fed T2 diet had the highest $(\mathrm{p}<0.05)$ total protein levels, whereas total protein was lowest $(\mathrm{p}<0.05)$ in $\mathrm{T} 1$ birds. Total bilirubin levels were highest $(\mathrm{p}<0.05)$ in T3 birds and lowest $(\mathrm{p}<0.05)$ in T1 and T2. T3 birds had the highest $(\mathrm{p}<0.05)$ Lactate dehydrogenase (LDH) levels, whereas LDH levels were lowest $(\mathrm{p}<0.05)$ in T1 birds. 
International Journal of Agriculture and Environmental Research

ISSN: 2455-6939

Volume: 07, Issue: 02 "March-April 2021"

Table 6: Blood parameters ofpre-starter local turkeys fed varying energy diets

\begin{tabular}{lllll}
\hline Parameters & T1 $(2600 \mathrm{Kcal} / \mathrm{kg} \mathrm{ME})$ & T2 $(2700 \mathrm{Kcal} / \mathrm{kg} \mathrm{ME})$ & T3 $(2800 \mathrm{Kca} / \mathrm{kg}$ ME $)$ & SEM \\
\hline PCV $\%$ & 40.67 & 38.67 & 43.00 & 1.59 \\
Hb Conc., g/dL & 11.72 & 12.42 & 11.59 & 0.35 \\
WBC, $\times 10^{9} / \mathrm{L}$ & 13.61 & 12.53 & 12.29 & 0.46 \\
RBC, $\times 10^{12} / \mathrm{L}$ & 4.24 & 4.17 & 4.22 & 0.06 \\
$\mathrm{MCH}, \%$ & 2.76 & 2.99 & 2.76 & 0.09 \\
MCHC, $\%$ & $29.05^{\mathrm{ab}}$ & $32.49^{\mathrm{a}}$ & $27.35^{\mathrm{b}}$ & 1.09 \\
Creatinine, mg/dL & $1.41^{\mathrm{a}}$ & $1.32^{\mathrm{b}}$ & $1.18^{\mathrm{c}}$ & 0.02 \\
Urea, $\mathrm{mg} / \mathrm{dL}$ & $37.35^{\mathrm{a}}$ & $33.37^{\mathrm{b}}$ & $32.20^{\mathrm{c}}$ & 0.09 \\
AST, U/L & $10.44^{\mathrm{b}}$ & $13.11^{\mathrm{a}}$ & $13.33^{\mathrm{a}}$ & 0.28 \\
ALT, U/L & $4.67^{\mathrm{b}}$ & $7.33^{\mathrm{a}}$ & $6.44^{\mathrm{a}}$ & 0.27 \\
TP, $\mathrm{mg} / \mathrm{dL}$ & $1.17^{\mathrm{c}}$ & $1.59^{\mathrm{a}}$ & $1.48^{\mathrm{b}}$ & 0.02 \\
TBIL, mg/dL & $14.41^{\mathrm{b}}$ & $14.62^{\mathrm{b}}$ & $15.43^{\mathrm{a}}$ & 0.07 \\
LDH, U/L & $43.53^{\mathrm{c}}$ & $45.56^{\mathrm{b}}$ & $46.48^{\mathrm{a}}$ & 0.09 \\
\hline
\end{tabular}

a,b, c Means within columns with different superscripts differ significantly (P<0.05); PCV: Packed cell volume; Conc: Concentration; RBC: Red blood cell; WBC: White blood cell; MCH: Mean cell haemoglobin: MCHC: Mean cell haemoglobin concentration; AST: Aspartate transaminase; ALT: Alanine amino transaminase; TP: Total protein; TBIL: Total bilirubin; LDH; Lactate dehydrogenase; SEM: Standard error of mean.

\section{Effect of dietary protein levels on haematological and serum-biochemical indices}

The effect of dietary supplementation of varying dietary levels of protein on the haematological and serum-biochemical indices of local turkeys at the pre- starter phase is shown in Table 7. T2 (26\% CP) birds had the highest $(\mathrm{p}<0.05)$ haemoglobin concentration $(\mathrm{Hb}), \mathrm{MCH}$ and $\mathrm{MCHC}$ compared with those fed the T1 $(24 \% \mathrm{CP})$ and T3 (28\% CP) diets. Urea, AST and LDH levels were highest $(\mathrm{p}<0.05)$ in T1 birds and lowest in T3. Birds fed T3 diet had the highest $(\mathrm{p}<0.05)$ total protein, while those fed $\mathrm{T} 2$ had the lowest $(\mathrm{p}<0.05)$ total protein levels. Total bilirubin values were highest $(\mathrm{p}<0.05)$ in T2 birds and lowest $(\mathrm{p}<0.05)$ in T1 birds. 
International Journal of Agriculture and Environmental Research

ISSN: 2455-6939

Volume: 07, Issue: 02 "March-April 2021"

Table 7: Blood parameters of pre-starter local turkeys fed varying protein diets

\begin{tabular}{|c|c|c|c|c|}
\hline Parameters & $\mathrm{T} 1(24 \% \mathrm{CP})$ & $\mathrm{T} 2(26 \% \mathrm{CP})$ & T3 $(28 \% \mathrm{CP})$ & SEM \\
\hline PCV (\%) & 42.67 & 39.22 & 40.44 & 1.59 \\
\hline $\mathrm{Hb}$ concentration $(\mathrm{g} / \mathrm{dL})$ & $11.15^{\mathrm{b}}$ & $13.67^{\mathrm{a}}$ & $10.92^{\mathrm{b}}$ & 0.34 \\
\hline $\mathrm{WBC}\left(\times 10^{9} / \mathrm{L}\right)$ & 13.27 & 12.89 & 12.28 & 0.45 \\
\hline $\mathrm{RBC}\left(\times 10^{12} / \mathrm{L}\right)$ & 4.27 & 4.20 & 4.17 & 0.05 \\
\hline $\mathrm{MCH}(\%)$ & $2.62^{\mathrm{b}}$ & $3.26^{\mathrm{a}}$ & $2.62^{\mathrm{b}}$ & 0.09 \\
\hline $\mathrm{MCHC}(\%)$ & $29.42^{\mathrm{b}}$ & $35.18^{\mathrm{a}}$ & $27.29^{\mathrm{b}}$ & 1.09 \\
\hline Creatinine (mg/dL) & 1.32 & 1.28 & 1.30 & 0.02 \\
\hline Urea $(\mathrm{mg} / \mathrm{dL})$ & $37.18^{\mathrm{a}}$ & $33.32^{\mathrm{b}}$ & $32.42^{\mathrm{c}}$ & 0.08 \\
\hline AST (U/L) & $14.44^{\mathrm{a}}$ & $12.33^{\mathrm{b}}$ & $10.11^{\mathrm{c}}$ & 0.27 \\
\hline $\operatorname{ALT}(\mathrm{U} / \mathrm{L})$ & $5.56^{\mathrm{b}}$ & $5.78^{\mathrm{b}}$ & $7.11^{\mathrm{a}}$ & 0.27 \\
\hline Total protein $(\mathrm{mg} / \mathrm{dL})$ & $1.40^{\mathrm{b}}$ & $1.30^{\mathrm{c}}$ & $1.53^{\mathrm{a}}$ & 0.02 \\
\hline Total bilirubin $(\mathrm{mg} / \mathrm{dL})$ & $13.43^{\mathrm{c}}$ & $16.14^{\mathrm{a}}$ & $14.89^{\mathrm{b}}$ & 0.06 \\
\hline Lactate dehydrogenase (U/L) & $51.14^{\mathrm{a}}$ & $44.17^{\mathrm{b}}$ & $40.27^{\mathrm{c}}$ & 0.08 \\
\hline
\end{tabular}

\section{The interaction effect of varying energy and protein levels on haematology and serum}

The interaction effects of varying dietary inclusion of energy and protein levels on haematological and serum-biochemical indices of local turkeys at the pre- starter phase is shown in Table 8. The highest $(\mathrm{p}<0.05) \mathrm{Hb}, \mathrm{MCH}$ and $\mathrm{MCHC}$ values were recorded in $\mathrm{T} 5(26 \% \mathrm{CP}+$ $2700 \mathrm{ME})$ birds, while birds fed T1 $(24 \% \mathrm{CP}+2600 \mathrm{ME})$ had the highest $(\mathrm{p}<0.05)$ value for white blood cell count (WBC). MCH was lowest $(\mathrm{p}<0.05)$ in T1, T2 $(24 \% \mathrm{CP}+2700 \mathrm{ME})$, T3 $(24 \% \mathrm{CP}+2800 \mathrm{ME})$ and $\mathrm{T} 9(28 \% \mathrm{CP}+2800 \mathrm{ME})$ whereas birds fed $\mathrm{T} 3$ had the lowest $(\mathrm{p}<0.05)$ values for MCHC; while those fed T8 $(28 \% \mathrm{CP}+2700 \mathrm{ME})$ had the lowest $(\mathrm{p}<0.05)$ values for WBC. Birds fed T1 and T2 has the highest $(\mathrm{p}<0.05)$ creatinine and urea values, while these values were lowest $(\mathrm{p}<0.05)$ in T3 and T7 $(28 \% \mathrm{CP}+2600 \mathrm{ME})$ birds. Birds fed T1, T2 and T6 (26\% CP + $2800 \mathrm{ME})$ had the highest $(\mathrm{p}<0.05)$ values for AST, while those fed T4 (26\% 
International Journal of Agriculture and Environmental Research

ISSN: 2455-6939

Volume: 07, Issue: 02 "March-April 2021"

$\mathrm{CP}+2600 \mathrm{ME}), \mathrm{T} 7$ and $\mathrm{T} 8(28 \% \mathrm{CP}+2700 \mathrm{ME})$ had the lowest $(\mathrm{p}<0.05)$ AST. ALT was highest $(\mathrm{p}<0.05)$ in T5 and T9 birds and lowest $(\mathrm{p}<0.05)$ in birds fed T1.The highest $(\mathrm{p}<0.05)$ concentration of total protein was recorded for T9 birds and the lowest $(\mathrm{p}<0.05)$ for T3, T4, T6 and T8 birds. Birds fed T5, T6 and T9 had the highest $(\mathrm{p}<0.05)$ total bilirubin values while total bilirubin was lowest $(\mathrm{p}<0.05)$ in T2 birds. LDH was highest $(\mathrm{p}<0.05)$ in T3 birds and lowest $(\mathrm{p}<0.05)$ in $\mathrm{T} 4$ and $\mathrm{T} 9$.

Table 8: Serum indices of pre-starter local turkeys fed varying energy and protein diets

\begin{tabular}{lllllllllll}
\hline Parameters & T1 & T2 & T3 & T4 & T5 & T6 & T7 & T8 & T9 & SEM \\
\hline PCV (\%) & 42.33 & 37.67 & 48.00 & 39.67 & 37.00 & 41.00 & 40.00 & 41.33 & 40.00 & 2.76 \\
Hb (g/dL) & $11.38^{\mathrm{bc}}$ & $10.89^{\mathrm{bc}}$ & $11.17^{\mathrm{bc}}$ & $13.41^{\mathrm{ab}}$ & $14.79^{\mathrm{a}}$ & $12.81^{\mathrm{abc}}$ & $10.37^{\mathrm{c}}$ & $1158^{\mathrm{bc}}$ & $10.80^{\mathrm{bc}}$ & 0.61 \\
WBC $\left(\times 10^{9} / \mathrm{L}\right)$ & $15.23^{\mathrm{a}}$ & $12.00^{\mathrm{ab}}$ & $12.57^{\mathrm{ab}}$ & $12.63^{\mathrm{ab}}$ & $14.50^{\mathrm{ab}}$ & $11.53^{\mathrm{ab}}$ & $12.97^{\mathrm{ab}}$ & $11.10^{\mathrm{b}}$ & $12.77^{\mathrm{ab}}$ & 0.79 \\
RBC $\left(\times 10^{12} / \mathrm{L}\right)$ & 4.17 & 4.23 & 4.40 & 4.37 & 4.10 & 4.13 & 4.20 & 4.17 & 4.13 & 0.10 \\
MCH $(\%)$ & $2.75^{\mathrm{b}}$ & $2.57^{\mathrm{b}}$ & $2.55^{\mathrm{b}}$ & $3.07^{\mathrm{ab}}$ & $3.61^{\mathrm{a}}$ & $3.10^{\mathrm{ab}}$ & $2.47^{\mathrm{b}}$ & $2.78^{\mathrm{ab}}$ & $2.62^{\mathrm{b}}$ & 0.17 \\
MCHC $(\%)$ & $26.94^{\mathrm{bc}}$ & $28.99^{\mathrm{bc}}$ & $23.33^{\mathrm{c}}$ & $33.77^{\mathrm{ab}}$ & $40.05^{\mathrm{a}}$ & $31.17^{\mathrm{abc}}$ & $26.45^{\mathrm{bc}}$ & $28.42^{\mathrm{bc}}$ & $27.02^{\mathrm{bc}}$ & 1.90 \\
Creat. (mg/dL) & $1.62^{\mathrm{a}}$ & $1.20^{\mathrm{cd}}$ & $1.11^{\mathrm{d}}$ & $1.42^{\mathrm{b}}$ & $1.27^{\mathrm{c}}$ & $1.15^{\mathrm{cd}}$ & $1.14^{\mathrm{cd}}$ & $1.49^{\mathrm{b}}$ & $1.28^{\mathrm{c}}$ & 0.02 \\
Urea (mg/dL) & $47.69^{\mathrm{a}}$ & $33.15^{\mathrm{cd}}$ & $30.70^{\mathrm{e}}$ & $33.75^{\mathrm{bc}}$ & $34.42^{\mathrm{b}}$ & $31.78^{\mathrm{d}}$ & $30.60^{\mathrm{e}}$ & $32.53^{\mathrm{d}}$ & $34.13^{\mathrm{b}}$ & 0.15 \\
AST (U/L) & $15.00^{\mathrm{a}}$ & $16.00^{\mathrm{a}}$ & $12.33^{\mathrm{b}}$ & $7.00^{\mathrm{c}}$ & $14.33^{\mathrm{ab}}$ & $15.67^{\mathrm{a}}$ & $9.33^{\mathrm{c}}$ & $9.00^{\mathrm{c}}$ & $12.00^{\mathrm{b}}$ & 0.48 \\
ALT (U/L) & $4.00^{\mathrm{c}}$ & $6.67^{\mathrm{ab}}$ & $6.00^{\mathrm{bc}}$ & $4.33^{\mathrm{bc}}$ & $8.67^{\mathrm{a}}$ & $4.33^{\mathrm{bc}}$ & $5.67^{\mathrm{bc}}$ & $6.67^{\mathrm{ab}}$ & $9.00^{\mathrm{a}}$ & 0.47 \\
TP (mg/dL) & $1.16^{\mathrm{de}}$ & $1.73^{\mathrm{b}}$ & $1.32^{\mathrm{c}}$ & $1.08^{\mathrm{e}}$ & $1.71^{\mathrm{b}}$ & $1.11^{\mathrm{e}}$ & $1.27^{\mathrm{cd}}$ & $1.32^{\mathrm{c}}$ & $1.99^{\mathrm{a}}$ & 0.03 \\
TBL (mg/dL) & $12.90^{\mathrm{d}}$ & $14.24^{\mathrm{c}}$ & $13.16^{\mathrm{d}}$ & $15.16^{\mathrm{b}}$ & $16.45^{\mathrm{a}}$ & $16.82^{\mathrm{a}}$ & $15.18^{\mathrm{b}}$ & $13.19^{\mathrm{d}}$ & $16.31^{\mathrm{a}}$ & 0.11 \\
LDH (U/L) & $46.17^{\mathrm{d}}$ & $48.42^{\mathrm{c}}$ & $58.81^{\mathrm{a}}$ & $32.12^{\mathrm{f}}$ & $52.03^{\mathrm{b}}$ & $48.36^{\mathrm{c}}$ & $52.31^{\mathrm{b}}$ & $36.24^{\mathrm{e}}$ & $32.26^{\mathrm{f}}$ & 0.15
\end{tabular}

a,b, c Means within columns with different superscripts differ significantly (P<0.05); PCV: Packed cell volume; Conc: Concentration; RBC: Red blood cell; WBC: White blood cell; MCH: Mean cell haemoglobin: MCHC: Mean cell haemoglobin concentration; AST: Aspartate transaminase; ALT: Alanine aminotransaminase; TP: Total protein; TBIL: Total bilirubin; LDH; Lactate dehydrogenase;T1: 24\% CP+ 2600 ME; T2: 24\% CP+ $2700 \mathrm{ME} ; \mathrm{T} 3: 24 \% \mathrm{CP}+2800 \mathrm{ME} ; \mathrm{T} 4: 26 \% \mathrm{CP}+2600 \mathrm{ME}$; T5: 26\% CP+ $2700 \mathrm{ME}$; T6: 26\% CP+ 2800 ME; T7: 28\% CP+ 2600 ME; T8: 28\% CP+ $2700 \mathrm{ME}$; T9: $28 \% \mathrm{CP}+2800 \mathrm{M}$; SEM: Standard error of mean. 
International Journal of Agriculture and Environmental Research

ISSN: $2455-6939$

Volume: 07, Issue: 02 "March-April 2021"

\section{DISCUSSION}

\section{The effect of dietary energy levels on growth performance}

The results in Table 3 showed that birds fed $2600 \mathrm{Kcal} / \mathrm{kg}$ ME diet (T1) had the highest FBW, TWG and PER. The birds also consumed less feed to give an improved FCR. This result indicates that poults in their early life (1-28days) need more of higher proteins than higher energy to grow. This finding supports an earlier report (15) that Bile salt and lipase secretions might be the limiting factor for fat absorption in the post hatch period. The reason for this is might be that the digestive tract of the poults is not yet fully developed to utilize high dietary energy levels in the post-hatch period of their life. (16) made similar observations when they reported that the absorption of an unsaturated fatty acid (oleic acid), by itself is high in posthatch chicks; however, an increased intake of this lipid depressed the percentage of absorption. The present study contradicts the assertion that feed intake was not influenced by diet composition (15). Other studies found that feed intake was influenced by energy density in the feed $(16 ; 17)$. However, in these studies, the effect was studied over a longer period of time beyond the first days post-hatch. Factors like pellet size, hardness and palatability might play an important role in inducing quick and easy ingestion of feed. In the present study (Table 3), we observed that high dietary energy levels did not translate into higher growth rate, and this might be attributed to the immaturity of the digestive tract. Intestinal mucosa, crypth depth and villus height need to develop in time $(18 ; 19)$ as well as enzyme secretions like lipase, trypsin and amylase $(20 ; 21)$.

\section{The effect of dietaryprotein levels on growth performance}

Our results in Table 4 showed that that poults fed on diets of $26 \% \mathrm{CP}$ diet performed better in terms of FBW, TWG, ADWG, and FCR and PER. This suggests than poults need more of higher protein to grow in their early stage (1-28days) of life. The results also showed that increasing crude protein level more than $26 \%$ may not necessarily be profitable to the turkey farmer. This supports the findings of (22) who reported that increasing levels of dietary CP at high ambient temperature had no positive effect on the growth performance of broilers. Based on the observation made in our study, it may suffice to state that optimum performance in turkey poults cannot be achieved by following the NRC (1994) standard nutrient profile, particularly, under the high ambient temperature that is prevalent in Nigeria. The results of growth performance in the current study are in accordance with the results of (23), who reported that reducing the $\mathrm{CP}$ in the diets of broiler birds had no significant $(\mathrm{P}>0.05)$ effect on their live weight gain. (24) had earlier reported that during the hot humid season, higher dietary levels of protein can possibly cause discomfort in birds due to heat stress. 
International Journal of Agriculture and Environmental Research

ISSN: 2455-6939

Volume: 07, Issue: 02 "March-April 2021"

\section{The effect of dietaryenergy and protein levels on growth performance}

The present study showed that the interaction of protein (26\% CP) and energy (2600 and 2700 $\mathrm{Kcal} / \mathrm{kg} \mathrm{ME}$ ) gave better results in terms FBW, TWG, ADWG and FCR. This study disagrees with the findings of (25) who reported that dietary $\mathrm{CP}$ had non-significant $(\mathrm{P}>0.05)$ effect on the feed intake of birds when diets were with minimum energy difference or iso-energic. The influence of energy: protein ratio on the feed consumption or their interaction on feed intake was reported to be non-significant in broiler birds (26). The economic analyses of the present study are similar to findings of (24) who reported that the least feed cost per $\mathrm{kg}$ body weight was recorded in starter broiler birds fed diets containing $21 \% \mathrm{CP}$ and $3100 \mathrm{Kcal} / \mathrm{Kg} \mathrm{ME}$. Our results on improved growth performance are similar to findings of (27) who reported that during 0-6 week period, poults receiving higher protein (24, 26 and 28\%) and higher energy (2600, 2800 and $3100 \mathrm{Kcal} / \mathrm{kg} \mathrm{ME}$ ) had significantly higher body weight. The protein and energy interaction also had significant effect $(\mathrm{P}<0.05)$ on body weight gain. However, the FCR of the higher protein dietary groups were similar with those of birds fed the low protein of $24 \%$. This result is in agreement with the results of (28) who reported that turkey poults fed high protein in combination with low energy diets had improved growth in the post-hatch period. Other studies $(29 ; 30)$ showed that body weight gain in poults increased during the first weeks with increase in dietary protein levels.

The reason might be that chicks have a high protein requirement in order to develop specific tissues, especially the small intestines in the post-hatch period $(20 ; 31)$. In this study, high energy levels beyond $2700 \mathrm{ME}$ did not result in a higher growth rate, which might be due to the immaturity of the digestive tract. The intestinal mucosa, crypth depth and villus height need to develop in time $(18 ; 19)$, coupled with enzymatic secretions like lipase, trypsin and amylase $(20$; 21). The reports of (32) showed that birds fed 3040 and $3120 \mathrm{Kcal} / \mathrm{kg}$ diets had improved feed efficiency; however, their weight gain was not influenced. In a study using diets similar to those of the current study, (33) observed that an increase in energy level improved the weight gain and feed conversion of birds with no effect on the feed intake, while (34) reported a reduction in feed intake at higher dietary energy inclusion levels.

\section{The effect of dietary energy and protein levels and their interaction on blood indices}

Blood analysis is important in the detection of some changes in the health status of birds, and can thus be a useful aid in the diagnosis of diseases in birds (35). Our results in Table 8 showed that the concentrations of AST, ALT, total protein, total bilirubin and LDH were consistently lower in $\mathrm{T} 4(26 \% \mathrm{CP}+2600 \mathrm{Kcal} / \mathrm{kg} \mathrm{ME})$ diet indicating that liver and kidney functions were not impaired. Urea and creatinine concentrations were however lowest in T3 $(24 \% \mathrm{CP}+2800$ $\mathrm{Kcal} / \mathrm{kg} \mathrm{ME})$ and $\mathrm{T} 7(28 \% \mathrm{CP}+2600 \mathrm{Kcal} / \mathrm{kg} \mathrm{ME})$ diets. Increase in AST, ALT and bilirubin 
International Journal of Agriculture and Environmental Research

ISSN: 2455-6939

Volume: 07, Issue: 02 "March-April 2021"

concentrations is a reflection of hepatic cytolysis and necrosis $(36 ; 37)$. An earlier report had attributed the increase in total protein to pathologies, inflammation or substantial dehydration, while elevated LDH is an indication of Vitamin- $B_{12}$ deficiency $(38 ; 39)$. High creatinine levels signifies an impairment in kidney function, whereas, elevated urea serum shows poor protein utilization in a feed mixture, deficiency of exogenous amino acid, or occurrence of dehydration (38). The low WBC recorded in $\mathrm{T} 8(28 \% \mathrm{CP}+2700 \mathrm{Kcal} / \mathrm{kg} \mathrm{ME})$ birds compared with those in $\mathrm{T} 1$ is an indication that $\mathrm{T} 8$ birds were in better health condition than $\mathrm{T} 1(24 \% \mathrm{CP}+2600 \mathrm{Kcal} / \mathrm{kg}$ ME diet) birds (40). $\mathrm{Hb}, \mathrm{MCH}$ and $\mathrm{MCHC}$ were improved in T5 birds, suggesting an increased ability in these birds to withstand respiratory stress compared to birds in other treatment groups (41).Our results on $\mathrm{Hb}$ and WBC does not align with the findings of (42) and (43) who reported that there were no significant treatment effect in the $\mathrm{Hb}$ and $\mathrm{WBC}$ of birds. The value we recorded for total protein in this study is lower than those reported by (44). The authors reported higher total protein values in 4 month-old wild turkey poults. The urea values and AST values of this study did not also fall within the range reported by (45) for 4 month-old wild turkey poults. These differences could be attributable to dietary composition, or to the innate differences between domestic and wild poults.

\section{CONCLUSION}

The results obtained in this study indicate that feeding local turkey poults on $26 \%$ crude protein and $2600 \mathrm{Kcal} / \mathrm{kg} \mathrm{ME}$ diets improved growth performance and did not impair their health status.

\section{ACKNOWLEDGEMENT}

The authors are grateful to Tertiary Education Trust Fund (TEFund), Nigeria for sponsoring this research through the research project no. TETFUND/DESS/UN/NSUKKA/2017/RP/VOL1.

\section{LITERATURE CITED}

1. Schonfeldt, H.C., and N. G. Hall. 2012. Dietary protein quality and malnutrition in Africa. Brit.J Nutr. 108(Supp 2):S69-S76.Doi: 10.10171S0007114512002553.

2. WHO. (World Health Organization). Protein and amino acid requirements in human nutrition: Report of a joint WHO/FAO/UNU expert consultation. WHO technical report series. Genf: 2007. Vol. 935.

3. Le Bihan-Duval, E., M. Debut, C.M. Berri, N. Sellier, V. Sante-Lhoutellier, Y. Jego,C. Beaumont 2008. Chicken meat quality: genetic variability and relationship with growth muscle characteristics. BMC Genet. 9:53. Doi: 10.1186/1471-2156-9-53 
International Journal of Agriculture and Environmental Research

ISSN: 2455-6939

Volume: 07, Issue: 02 "March-April 2021"

4. Ewa, J. E., C. N. Onwusiribe, F. C. Nzeakor. 2017. Trends and determinants of price in the poultry sub-sector of Nigeria. Sci. Papers Series Mgt Econ. Eng. Agric. Rural Devt. $17: 1$.

5. Adene, D. F., and A. E. Oguntade. 2006. The structure and importance of the commercial and village base poultry industry in Nigeria. FA (Rome), Italy.

6. FAO, 2010. Food and Agricultural Organization. Poultry, meat and eggs. Agribusiness Handbook. Fao.org/3/al175e/al175e.pdf.

7. Mbanasor, J. A., A. Sampson, 2004. Socio-economic determinants of turkey production among Nigerian Soldiers. Int. J. Poult Sci. 3(8):497-502. Doi; 10.4314/dai.v16i3.15671.

8. Abeke, F. O., and E. O. A. Ubani. 2008. Turkey production with emphasis on poult management. p. 321-323. Proc. Anim. Sci. Assoc. Nig. ABU, Zaria, Nigeria.

9. National Research Council, 1994. Nutrient requirements of poultry. 9th revised ed. National Academy Press, Washington DC, USA.

10. BUT. (2005). Commercial Performance Goals, 5th ed. British United Turkeys Ltd., Warren Hall, Broughton, UK

11. Jain, N. C. 1993. Essential of Veterinary hematology. Lea and Febiger, Philadelphia, USA.

12. Meyer, D. J., E. H. Coles, and L. J. Rich. 1992. Veterinary Laboratory Medicine Interpretation and Diagnosis. Saunders, W. B, Philadelphia, USA.

13. Evans, G. O. 1996. Animal Clinical Chemistry: A primer for Toxicologist. Taylor and Francis, London.

14. SAS, 2010. The SAS system for windows. Statistical Analysis System Institute Inc. Users Guide; SAS: Carry, NC, USA.

15. Krogdahl, A., and J. L. Sell 1989. Influence of age on lipase, amylase, and protease activities in pancreatic tissue and intestinal contents of young turkeys. Poult Sci. 68(11):1561-1568. Doi: 10.3382/ps.0681561.

16. Noy, Y. and Sklan, D. 2002. Nutrient use in chicks during the first week posthatch. Poult. Sci. 81(3):391-399. Doi: 10.1093/ps/81.3.391. 
International Journal of Agriculture and Environmental Research

ISSN: 2455-6939

Volume: 07, Issue: 02 "March-April 2021"

17. Plavnik, I., E. Wax, D. Sklan, I. Bartov, and S. Hurwitz. 1997. The response of broiler chickens and turkey poults to dietary energy supplied either by fat or carbohydrates. Poult Sci. 76 (7):1000-1005.Doi: 10.1093/ps/76.7.1000.

18. Uni, Z., S. Ganot, D. Sklan. 1998. Posthatch development of mucosal function in the broiler small intestine.Poult Sci. 77(1):75-82. Doi:10.1093/ps/77.1.75.

19. Yi, G.F., G. L. Allee, C. D. Knight, J. J. Dibner. 2005. Impact of glutamine and oasis hatchling supplement on growth performance, small intestinal morphology, and immune response of B. V. broiler vaccinated and challenged with Eimeria maxima. Poult Sci. 84(2): 283-293.Doi:10.1093/ps/84.2.283

20. Nitsan, Z., G. Ben-Avraham,Z. Zipora, and I. Nir. 1991. Growth and development of the digestive organs and some enzymes in broiler chicks after hatching. Br. Poult. Sci. 32(3): 515-523. Doi: 10.1080/00071669108417376.

21. Sklan, D. and Noy, Y. 2000. Hydrolysis and absorption in the small intestines of posthatch chicks. Poult. Sci. 79(9):1306-1310. Doi:10.1093/ps/79.9.1306.

22. Ojano-Dirain, C.P. and P.W. Waldroup. 2002. Protein and amino acid needs of broilers in warm weather: A review. Int. J. Poult. Sci. 1: 40-46.Doi:10.3923/ijps.2002.40.46.

23. Oyedeji, J. O., J. O. Umaigba, O. T. Okugbo, and P. A. Ekunwe. 2005. Response of broiler chickens to different dietary crude protein and feeding regimens. Rev. Bras. Cienc. Avic. 7(3):165-168. Doi: 10.1590/S1516-635X200500300005.

24. Siddiqur-Rehman, M., M.A.H. Pramanik, B. Basak, S.U. Tarafdar and S.K. Biswas. 2002. Effect of feeding low protein diet on performance of broiler during hot-humid season. Int. J. Poult. Sci. 1:35-39. Doi:10.3923/ijps.2002.35.39.

25. Mbajiorgu, C.A., J.W. Ngambi and D. Norris. 2011. Effect of varying dietary energy to protein ratio level on growth and productivity of indigenous Venda chickens. Asian J. Anim. Vet. Adv. 6: 344-352. Doi: 10.3923/ajava.2011.344.352.

26. Summers, J.D., D. Sprat, and J. L. Atkinson. 1992. Broiler weight gain and carcass composition when fed diets varying in amino acid balance, dietary energy and protein level. Poult. Sci. 71(2):263-273. Doi:10.3382/ps.0710263.

27. Majumdar, S., T. Praveenk, S. K. Bhanja, S. K. Agarwal, R. P. Singh. 2002. Growth performance and carcass characteristics of turkey poults maintained at different protein and energy levels during winter. Indian J Poult. Sci. 37:236-240. 
International Journal of Agriculture and Environmental Research

ISSN: 2455-6939

Volume: 07, Issue: 02 "March-April 2021"

28. Molenar, R., I.A.M. Reijrink, R. Meijerhof, J.K.W.M. Sparla and P.J. A. Wijtten. 2009. Effect of protein and energy level in feed on post hatch chick performance. Anim. Prod Sci. 56(11):1857-1866.

29. Sklan, D. and Noy, Y. 2003. Crude protein and essential amino acid requirements in chicks during the first week posthatch. Brit. Poult Sci. 44(2):266274.Doi:10.1080/10007166031000124586.

30. Wijtten, P. J. A., A. Lemme, D. J. Langhout. 2004. Effects of different dietary ideal protein levels on male and female broiler performance during different phases of life: Single phase effects, carryover effects and interactions between phases. Poult. Sci. 83(12):2005-2015.Doi:10.1093/ps/83.12.2005.

31. Sklan, D. 2001. Development of the digestive tract in poultry. World's Poult Sci. J. 57(4): 415-428. Doi:10.1079/WPS20010030.

32. Infante-Rodríguez, F., J. Salinas-Chavira, M. F. Montaño-Gómezet al.2016. Effect of diets with different energy concentrations on growth performance, carcass characteristics and meat chemical composition of broiler chickens in dry tropics. SpringerPlus.5:1937https://doi.org/10.1186/s40064-016-3608-0.

33. Tancharoenrat, P., and V. Ravindran2014. Influence of tallow and calcium concentrations on the performance and energy and nutrient utilization in broiler starters. Poult Sci. 93(6):1453-1462.Doi:10.3382/ps.2013-03817.

34. Kim, J.S., J. T. Kwon, L. Harim, J. H. Kim, S. K. Oh, B. K. Lee, L. Zheng, M. S. Konkuk Jung, B. K. An, C. W. Kan. 2012. Performance and carcass characteristics of two different broiler strains by different levels of metabolizable energy. Korean J Poult Sci. 39:195-205. Doi: 10.5536/KJPS.2012.39.3.195.

35. Toghyani, M., M. Toghyani, A. A. Gheisari, G. Ghalamkari, and S. Eghbalsaeid. 2011 Evaluation of cinnamon and garlic as antibiotic growth promoter substitutions on performance, immune responses, serum biochemical and haematological parameters. Livest Sci. 138 (1-3):167-173.Doi:10.1061/j.livsci.2010.12.018.

36. Zuonongo, M. Z. 2013. Determination of usual biochemical parameters in small ruminants in Burkina Faso and their variation in naturally infected subjects by trypanosomiasis. [Thesis for the rank of veterinary doctor], Ecole interetats des sciences et medecine veterinaire. 
37. Alabi, Q. K., R. O. Akomolafe,O. S. Olukiran, A. O. Nafiu, J. G. Omole, A. M. Adefisayo, A. A. Oladele. 2017. Assessment of haematological and biochemical effects of Kolaviron in male Wister rats. Bri. J Pharm. Res.16(3):1-14.

38. Kaneko, J. 2008. Clinical biochemistry of domestic animals. Academic Press, San Diego, California.

39. Krauze, M., and E. R. Grela. 2010. Effects of an Alfafa concentrate in turkey diets on performance and some blood parameters. Arch. Geflugelk. 74 (4):S.226-232.

40. Grela, E. R., A. Brodacki, J. Batkowska, J. Matras. 2009. Influence of a probiotic Bacillus toyoi strain on performance of growing turkey poults. Arch. Geflugelk. 73(10):S.160-166.

41. Afolabi, K. D., A. O. Akinsoyinu, A. R. O. Abdullah, R. Olajide, and S. B. Akinleye 2011. Haematological parameters of the Nigerian local grower chickens fed varying dietary levels of palm kernel cake. Poljoprivreda. 17(1):74-78.

42. Bounous, D.I., R.D. Wyatt, P.S. Gibbs, J.V. Kilburn and C.F. Quist. 2000. Normal hematologic and serum biochemical reference intervals for juvenile wild turkeys. J. Wildlife Dis. 36(2): 393-396. Doi: 10.7589/0090-3558-36.2.393.

43. Elizabeth, M. S. S, C. P. Antonio, R. V. M. Gislaine, M. L. Ivan, J. P. T. Alan, N. Leonildo, D. Janine, and J. F. José. 2009. Haematology of the Bronze Turkey (Meleagris Gallopavo): variations with age and gender. Int. J Poult Sci. 8 (8):752-754. 10.3923/ijps.2009.752.7543.

44. Denise I. Bounous, Roger D. Wyatt, Penelope S Gibbs, J.V. Kilburn and Charlotte F. Quist. Normal Hematologic and Serum Biochemical Reference Intervals for Juvenile Wild Turkeys. May 2000: Journal of wildlife diseases 36(2):393-6 DOI: 10.7589/00903558-36.2.393 